\title{
A URGÊNCIA NO ATENDIMENTO AO IDOSO: ESTUDO DE CASO
}

\author{
THE URGENCY IN ELDERLY CARE: A CASE STUDY
}

\section{Ana Paula dos Santos Zepka ${ }^{1}$ Thiago Leite Caetano²}

Resumo: O objetivo deste estudo foi caracterizar o perfil epidemiológico do idoso que busca consulta médica em Unidade de Pronto Atendimento. A amostra foi composta por 2273 pacientes, com idade acima de 60 anos atendidos em São José dos Campos, São Paulo, durante o ano de 2013. Os pacientes foram divididos em dez grupos de acordo com o diagnóstico, baseado na CID-10. As mulheres representaram a maioria dos pacientes (59\%). Do total de idosos, cerca de $24 \%$ foram diagnosticados como grupo $X$ (doenças variadas e não graves), demonstrando que um quarto da amostra foi de pacientes não urgentes, os quais poderiam ter sido consultados em outros serviços de saúde, ao invés do Pronto Atendimento. O conhecimento sobre o idoso que busca o serviço de urgência é fundamental para a orientação desse usuário ao local mais adequado para o tratamento. Com o aumento da expectativa de vida, é imprescindível a implementação de políticas públicas direcionadas ao idoso. $O$ cuidado com esse paciente é incipiente, pois os serviços de saúde ainda necessitam ser estruturados de acordo com as necessidades específicas desse público.

Palavras-chave: idoso; medicina de emergência; CID.

Abstract: The aim of this study was to characterize the profile of the elderly seeking medical consultation in an Emergency Unit. The sample comprised 2273 subjects over 60 years old, who were attended in São José dos Campos, São Paulo, during the year 2013. The patients were divided into ten groups according to the diagnosis based on ICD-10. Women accounted for the majority of patients (59\%). About $24 \%$ was diagnosed as $X$ group (varied and non-serious diseases), showing that a quarter of the sample was non-urgent patients, which could have been attended at other health facilities, rather than the Emergency Unit. Knowledge about the elderly who seeks emergency service is critical to guide them to the most appropriate place for treatment. With the increase in life expectancy, it is essential to implement public policies directed at the elderly. The care of this patient is incipient, as health services still need to be structured according to the specific needs of this public.

Keywords: elderly; emergency medicine; ICD.

\footnotetext{
${ }^{1}$ Doutora em Ciências - Instituto Nacional de Pesquisas Espaciais - Inpe, Brasil. Bióloga - Unimed Cooperativa de Trabalho Médico, São José dos Campos, SP - UNIMED. E-mail: anazepka@gmail.com.

2 Pós-graduado em Geriatria e Gerontologia - Faculdade de Ciências Médicas de Minas Gerais - FCMMG, Brasil. Médico

- UNIMED. E-mail: drthiagogeriatria@gmail.com.
}

Revista Univap - revista.univap.br

São José dos Campos-SP-Brasil, v. 21, n. 37, jul.2015. ISSN 2237-1753 


\section{INTRODUÇÃO}

O homem está vivendo cada vez mais. O envelhecimento é reflexo das melhorias no acesso à saúde, diminuição na taxa de mortalidade infantil, declínio da fecundidade e queda na mortalidade das partunientes (RODRIGUES et al., 2007). No Brasil, a expectativa de vida é de 74,6 anos, segundo o Instituto Brasileiro de Geografia e Estatística (2013). Projeções até 2050 (VERAS, 2012) estimam que a porcentagem de jovens continue a declinar até a maior concentração na população idosa, intensificando o envelhecimento demográfico nacional.

Segundo o Estatuto do Idoso (Lei 10741/03), o paciente acima de 60 anos deve ser atendido por pessoas devidamente treinadas para lidar com idosos (BRASIL, 2003). A geriatria é a especialidade médica apta a tratar da velhice, pois compreende a prevenção e o manejo das doenças do envelhecimento (VERAS; LOURENÇO, 2006). O envelhecimento está relacionado à diminuição da capacidade funcional em relação às doenças físicas, mas também às demências e depressão que geram limitações significativas ao indivíduo, comprometendo a qualidade de vida e a convivência familiar. Aliado ao envelhecimento, há o surgimento de doenças crônicas, como hipertensão arterial e doenças articulares, as quais juntamente com maus hábitos de saúde, contribuem para a incapacitação do idoso (FANHANI et al., 2007).

A modificação da estrutura etária da população foi acontecendo sem o devido acompanhamento de políticas públicas adequadas, com despreparo no acolhimento do idoso pelo sistema de saúde e, também, pelo cuidador e família. A educação dos pacientes e seus familiares é um fator importante para o conhecimento das doenças e consequente procura pela adequada assistência (LUNDELIN et al., 2012).

O atendimento ao idoso demanda profissionais especializados no tratamento desse paciente, garantindo a melhoria na saúde e consequente diminuição das visitas ao hospital. As Unidades de Pronto Atendimento, estruturadas para captar pacientes urgentes, são comumente procuradas pelos idosos em quaisquer circunstâncias. Atendimento "não urgente" é aquele em que o grau de severidade de um problema médico é baixo, mas, mesmo assim, resulta na busca do paciente pelo serviço de urgência (DURAND et al., 2011a). Extrapolar a demanda de pacientes compromete a qualidade do atendimento, acresce custos à instituição de saúde e expõe o idoso a um ambiente insalubre. Segundo o Ministério da Saúde, 65\% dos casos atendidos nos setores de urgência deveriam ser resolvidos na rede de atenção básica de saúde, como as Unidades Básicas de Saúde (UBS) e Unidades Especializadas (UES) (BRASIL, 2006).

O presente estudo teve por objetivo delinear o perfil epidemiológico do idoso que busca o Pronto Atendimento. Classificaram-se os pacientes em dez grupos, de acordo com as doenças diagnosticadas. Buscou-se, também, debater a visita do idoso como fator agravante na superlotação do hospital e investigar a possível sazonalidade na frequência das consultas efetuadas. 


\section{MATERIAL E MÉTODO}

Estudo quantitativo sobre os idosos assistidos por um único médico em um Pronto Atendimento da cidade de São José dos Campos, interior do estado de São Paulo, Brasil, durante o período de janeiro a dezembro de 2013. Foram analisadas as variáveis: gênero, idade, quantidade de pacientes, doença, segundo a Classificação Estatística Internacional de Doenças e Problemas Relacionados à Saúde - Décima Revisão (CID-10) e data da consulta. Dividiram-se os idosos em três faixas etárias definidas pela Organização Mundial da Saúde (OMS): de 60 a 69 anos, 70 a 79 anos e 80 anos ou mais.

Foi empregada a estatística por meio da média, desvio padrão e proporção (\%), além do teste t, para avaliar a normalidade de distribuição das variáveis. A correlação entre as variáveis numéricas foi investigada pelo cálculo do coeficiente de correlação de Pearson ( $r$ ). Valores de $p<0,05$ foram considerados, estatisticamente, significativos. Foi analisado o grau de dependência entre as variáveis, conforme interpretação de Dancey e Reidy (2006), que determinaram três grupos baseados na magnitude de " $r$ ": o de relação fraca ( $r=0,10$ até 0,30$)$, de relação moderada $(r=0,40$ até 0,60$)$ e de relação forte $(r=0,70$ até 1$)$.

Foram considerados dez grupos classificatórios, de acordo com as doenças diagnosticadas, conforme segue: I - doenças cardiovasculares, II - doenças respiratórias, III - doenças gastrointestinais, IV - doenças do trato geniturinário, V - doenças dermatológicas, VI - doenças endócrinas, VII - doenças psiquiátricas/ neurológicas, VIII - doenças traumatológicas, IX - doenças oncológicas e X - outras doenças.

Foram obedecidas as diretrizes éticas para pesquisas envolvendo seres humanos, de acordo com a Resolução no 196/96 do Conselho Nacional de Saúde (BRASIL, 1996).

Por se tratar de um estudo que analisa dados obtidos de um programa de gestão hospitalar, este trabalho não foi submetido a um Comitê de Ética em Pesquisa com Seres Humanos.

\section{RESULTADOS E DISCUSSÃO}

Dos 9111 atendimentos realizados de janeiro a dezembro de 2013, 2273 (25\%) foram de idosos. Desses, a maioria foi representada por pacientes do sexo feminino $(n=1337,59 \%)$. Outros autores já observaram que as mulheres acima de 60 anos tendem a utilizar mais os serviços de saúde do que os homens (OLIVEIRA et al., 2011; SILVEIRA; SANTOS; FERREIRA, 2012). 
Gráfico 1 - Número total de pacientes ( $\mathrm{n}$ ) para cada gênero (feminino: M e masculino: H), consultados em um Pronto Atendimento de São José dos Campos/ SP, em 2013, considerando a idade média em cada faixa etária

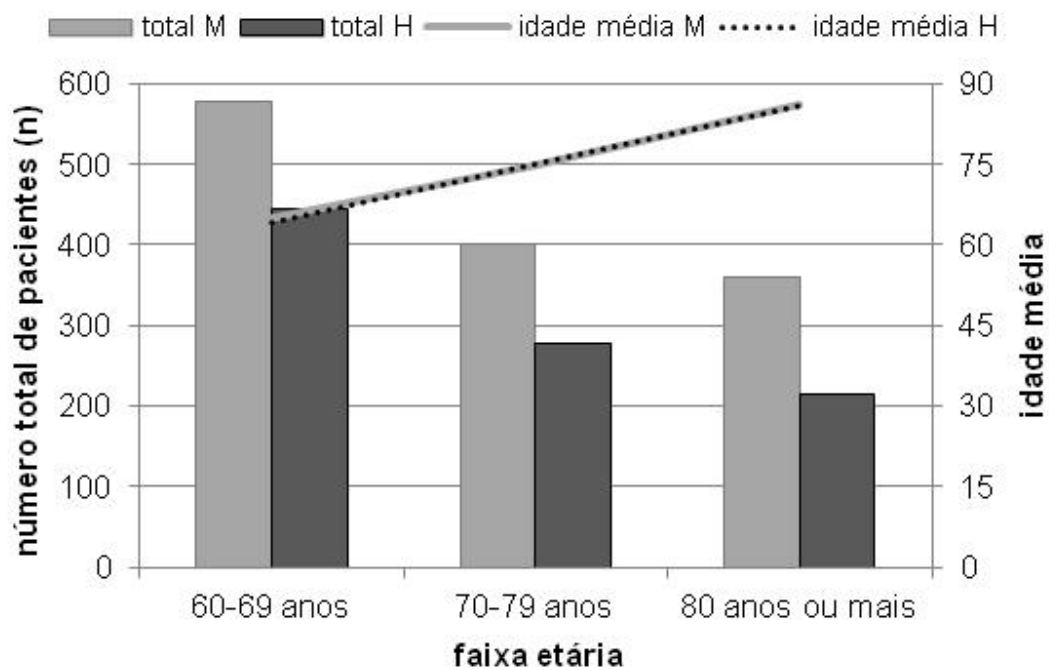

No Gráfico 1, observa-se que a quantidade atendida de mulheres foi maior nas três faixas etárias. Conforme o aumento da idade, o número de pacientes de ambos os sexos foi diminuindo e a quantidade de homens se mantendo menor em relação à de mulheres $(23 \%$, $31 \%$ e $40 \%$ a menos, respectivamente para cada categoria etária). Foi evidenciada a maior longevidade das mulheres, especialmente na faixa etária mais avançada ( 80 anos ou mais).

Moreira (1997) e Nascimento (2001) destacam a feminização do envelhecimento populacional como uma diferença de mortalidade, entre homens e mulheres que tende a se ampliar nos grupos etários mais avançados. Sabe-se que, culturalmente, as mulheres buscam mais assistência médica ao longo da vida e têm maior preocupação com a própria saúde, investindo em prevenção e autocuidado, o que colabora para o tratamento precoce de doenças e, consequentemente, contribui para a maior expectativa de vida (SILVEIRA et al., 2013). Além disso, as mulheres vivem mais devido a um conjunto de fatores, tais como: maior proteção cardiovascular em função dos hormônios, melhor sociabilidade e menor consumo de álcool e tabaco. Aquino, Menezes e Amoedo (1992) e Simons (2008) também observaram ser a demanda feminina por cuidados de saúde e assistência médica maior do que a masculina.

$\mathrm{Na}$ divisão dos idosos em dez grupos classificatórios, de acordo com as doenças diagnosticadas (Tabela 2), pode-se perceber que somente nos grupos VI (doenças endócrinas) e IX (doenças oncológicas) a quantidade de homens foi maior do que a de mulheres (12 contra 5 e 13 contra 7, respectivamente). As doenças cardiovasculares, significativas ao gênero masculino, acometeram 151 homens e 164 mulheres, todavia, estatisticamente, como o total de pacientes do sexo masculino foi menor (936), no grupo I a porcentagem de homens foi mais significativa, isto é, $16,1 \%$ contra $12,3 \%$ do sexo feminino. 
Tabela 1 - Classificação dos idosos consultados em um Pronto Atendimento de São José dos Campos/ SP, em 2013, em dez grupos distintos de doenças $(n=x)$

\begin{tabular}{ccccccc}
\hline \multirow{2}{*}{ Grupo } & \multicolumn{2}{c}{ Mulheres } & \multicolumn{2}{c}{ Homens } & \multicolumn{2}{c}{ Total } \\
& $\mathbf{n}$ & $\%$ & $\mathbf{n}$ & $\%$ & $\mathbf{n}$ & $\%$ \\
\hline I & 164 & 12,3 & 151 & 16,1 & 315 & 13,9 \\
II & 380 & 28,4 & 245 & 26,1 & 625 & 27,5 \\
III & 201 & 15,0 & 113 & 12,1 & 314 & 13,8 \\
IV & 99 & 7,4 & 47 & 5,1 & 146 & 6,4 \\
V & 62 & 4,6 & 41 & 4,4 & 103 & 4,5 \\
VI & 5 & 0,4 & 12 & 1,3 & 17 & 0,8 \\
VII & 41 & 3,1 & 13 & 1,4 & 54 & 2,4 \\
VIII & 76 & 5,7 & 56 & 6,0 & 132 & 5,8 \\
IX & 7 & 0,5 & 13 & 1,4 & 20 & 0,9 \\
X & 302 & 22,6 & 245 & 26,1 & 547 & 24,0 \\
Total & 1337 & 100,0 & 936 & 100,0 & 2273 & 100,0 \\
\hline
\end{tabular}

As doenças cardiovasculares são a principal causa de mortes em todo o mundo, sendo responsáveis por 30\% do número total, anualmente (MENDIS; PUSKA; NORRVING, 2011). Estimativas de 2010 demonstraram que países em desenvolvimento foram os mais afetados, sendo responsáveis por $80 \%$ das mortes (GARCÍA; ARTERO, 2011). Programas de prevenção às doenças cardiovasculares são implementados em países da América Latina, como Argentina, Chile e Brasil (PAN AMERICAN HEALTH ORGANIZATION, 2011) por meio de políticas públicas de conscientização sobre o controle da pressão arterial e contra o uso do tabaco. Essas são iniciativas úteis com importantes impactos sociais e econômicos, que buscam minimizar as despesas com saúde e as perdas em anos produtivos devido à mortalidade prematura, especialmente dos homens (BRASIL, 2011). Homens apresentam maior prevalência de fatores de risco, tais como: índice de massa corporal $(I M C)>25$, tabagismo, consumo de bebida alcoólica, sedentarismo e hipertensão, o que favorece a maior incidência de doenças cardiovasculares (MENDIS; PUSKA; NORRVING, 2011; CONCEIÇÃO et al., 2006; SOUZA et al., 2012), conforme também identificado neste estudo.

A análise da Tabela 1 permitiu identificar que as idosas foram diagnosticadas, em sua maioria (28,4\%), com doenças do grupo II (respiratórias), assim como os idosos, porém, esses em menor proporção $(26,1 \%)$. As doenças respiratórias são significativas aos idosos, constituindo importante causa de internação e óbito, principalmente naqueles pacientes em faixa etária mais avançada (FRANCISCO; DONALISIO; LATTORRE, 2004). Para Siqueira et al. (2004), quando expostos a hospitais, os pacientes com 60 anos ou mais tornam-se suscetíveis às enfermidades oportunistas, especialmente as respiratórias, que são agravadas quanto maior for a frequência das visitas.

Os homens, coincidentemente, também em 26,1\% apresentaram doenças categorizadas no grupo $X$ (outras). Aproximadamente, um quarto dos idosos (24\%) investigados neste trabalho foi diagnosticado como grupo $X$, que reúne doenças variadas $e$ comumente não graves. Esses dados remetem ao grande contingente de pacientes não urgentes que são, rotineiramente, consultados no Pronto Atendimento, superlotando-o. 
A desestruturação dos serviços de saúde dificulta o atendimento ao usuário para as unidades básicas ou especializadas, de modo que a queixa aguda é encaminhada ao Pronto Atendimento que, muitas vezes, considera as reclamações como não urgentes e condizentes ao perfil das unidades ambulatoriais, sendo para lá remanejadas. Segundo Durand et al. (2011a), os pacientes não urgentes são definidos como aqueles que poderiam ser tratados em ambulatório.

A superlotação dos hospitais está relacionada, dentre outras questões, ao excesso de pacientes não urgentes, o que poderia ser minimizado com a devida triagem dos usuários para a correta determinação da prioridade de tratamento (DURAND et al., 2011b). Entretanto, conforme Gentile et al. (2010), não existe um método consensual de triagem, que seja confiável e reprodutível. As avaliações usadas como referência para recusa de atendimento a pacientes potencialmente não urgentes remetem às questões legais, éticas e de segurança a considerar, o que exige cautela do sistema de saúde ao usar critérios restritivos no acesso dos usuários ao Pronto Atendimento.

Quanto às doenças menos incidentes, homens e mulheres apresentaram comportamento similar, demonstrando menor quantidade de atendimentos nos mesmos grupos (doenças endócrinas, outras doenças, doenças psiquiátricas/ neurológicas e doenças dermatológicas), apesar da diferença no número de pacientes para ambos os sexos. Considerando o total de idosos, o grupo VI deteve a menor proporção de pacientes $(0,8 \%)$, isto é, a amostra concentrou poucos pacientes com queixas relacionadas à diabetes, mesmo sendo eles insulino-dependentes ou não. Acredita-se que isso possa ter ocorrido em virtude desses usuários já apresentarem a doença sob o controle de medicamentos ou, ainda, por desconhecerem o problema.

Tabela 2 - Correlação de Pearson sobre a relação dos meses com os dez grupos classificatórios de doenças diagnosticadas em idosos consultados em um Pronto Atendimento de São José dos Campos/ SP, em 2013

\begin{tabular}{ccccccc}
\hline \multirow{2}{*}{ Meses } & \multicolumn{3}{c}{ Mulheres } & \multicolumn{3}{c}{ Homens } \\
\cline { 2 - 7 } & $\mathbf{n}^{\star}$ & $\mathbf{r}^{\star \star}$ & $\mathbf{p}^{\star \star \star}$ & $\mathbf{n}^{*}$ & $\mathbf{r}^{\star \star}$ & $\mathbf{p}^{\star \star \star}$ \\
\hline Janeiro & 49 & $-0,239$ & 0,765 & 58 & $-0,170$ & 0,895 \\
Fevereiro & 36 & $-0,333$ & 0,275 & 28 & $-0,022$ & 0,093 \\
Março & 116 & $-0,374$ & 0,109 & 67 & $-0,284$ & 0,629 \\
Abril & 157 & $-0,273$ & 0,088 & 110 & $-0,268$ & 0,194 \\
Maio & 163 & $-0,381$ & 0,100 & 100 & $-0,272$ & 0,290 \\
Junho & 137 & $-0,395$ & 0,139 & 71 & $-0,499$ & 0,540 \\
Julho & 137 & $-0,475$ & 0,197 & 90 & $+0,032$ & 0,328 \\
Agosto & 102 & $-0,397$ & 0,297 & 111 & $-0,398$ & 0,259 \\
Setembro & 85 & $-0,002$ & 0,318 & 75 & $-0,011$ & 0,496 \\
Outubro & 120 & $-0,079$ & 0,167 & 71 & $-0,359$ & 0,537 \\
Novembro & 106 & $-0,228$ & 0,168 & 72 & $-0,180$ & 0,501 \\
Dezembro & 129 & $-0,221$ & 0,085 & 83 & $-0,182$ & 0,370 \\
\hline${ }^{*}$ Quantidade de pacientes; ${ }^{* *}$ valor da relação; ${ }^{* * *}$ Correlação é significante ao nível $p<0,05$.
\end{tabular}


Conforme Tabela 2, não foram observadas correlações significativas entre as variáveis, sendo $p>0,05$ e próximo de zero, isto é, não ocorreu linearidade entre o número de pacientes (mulheres e homens) que visitaram o Pronto Atendimento e o mês em que a consulta foi realizada. Quanto mais próximo o valor de "r" está do zero (independente do sinal) menor é a força da relação entre as variáveis. Neste estudo, segundo interpretação de Dancey e Reidy (2006), em 92\% dos casos, as relações foram consideradas fracas.

Apesar de não ter sido evidenciada sazonalidade na visita do idoso ao Pronto Atendimento, com maior ou menor frequência de consultas ao longo do ano, quando se analisa a correlação de Pearson, destaca-se que, para as mulheres a quantidade máxima de atendimentos ocorreu em abril e maio (outono), quando a maioria das idosas (33\%) foi acometida por enfermidades respiratórias (grupo II) e 21\%, por outras doenças (grupo X). Nos homens, os picos de atendimento foram em abril (outono) e agosto (inverno) nos quais 33\% e $23 \%$ dos idosos foram diagnosticados com doenças dos grupos II e X, respectivamente. Nessas estações, a temperatura e umidade relativa do ar são mais baixas, o que contribui para o agravamento de doenças respiratórias, como gripes e pneumonias, especialmente em idosos já debilitados. Rodrigues et al. (2010), também, observaram que durante o período frio e seco, as taxas de internação de idosos, por asma e outros problemas respiratórios, são mais elevadas.

\section{CONCLUSÃO}

Com o aumento da expectativa de vida é imprescindível a formulação e implementação de políticas públicas efetivas, direcionadas ao idoso. O Pronto Atendimento, comumente procurado por idosos para resolução imediata dos problemas de saúde, necessita ser adaptado e estruturado a esse público.

Neste estudo, foi utilizada uma classificação de dez grupos para as doenças diagnosticadas. Os resultados demonstraram que alguns pacientes poderiam ter sido consultados em outros serviços da rede básica de saúde, ao invés do Pronto Atendimento, que representa ao usuário a alternativa mais fácil e rápida, em caso de doença.

O conhecimento do perfil do idoso é fundamental tanto para subsidiar o atendimento no serviço de urgência como para orientar o usuário ao local mais apropriado para tratamento, resolvendo os problemas de maneira mais eficaz e evitando exposição desnecessária do paciente ao Pronto Atendimento. A solução é a informação e o melhor reconhecimento do público alvo e sua real necessidade.

\section{REFERÊNCIAS}

AQUINO, E. M. L.; MENEZES, G. M. S.; AMOEDO, M. B. Gênero e saúde no Brasil: considerações a partir da Pesquisa Nacional por Amostra de Domicílios. Revista de Saúde Pública, São Paulo, v. 26, n. 3, p. 195-202, jun., 1992. 
BRASIL. Resolução no 196 de 10 de outubro de 1996. Diretrizes e normas regulamentadoras de pesquisa envolvendo seres humanos. Brasília: Diário Oficial da União, 1996.

Lei $n^{\circ} 10741$ de 01 de outubro de 2003. Dispõe sobre o Estatuto do Idoso e dá outras providências. Brasília: Diário Oficial da União, 2003.

Política Nacional de Atenção às urgências. Brasília: Editora do Ministério da Saúde, 2006.

Vigitel Brasil 2010: vigilância de fatores de risco e proteção para doenças crônicas por inquérito telefônico. Brasília: Editora do Ministério da Saúde, 2011.

CONCEIÇÃO, T. V. et al. Valores de pressão arterial e suas associações com fatores de risco cardiovasculares em servidores da Universidade de Brasília. Arquivos Brasileiros de Cardiologia, São Paulo, v. 86, n. 1, p. 26-31, jan. 2006.

DANCEY, C.; REIDY, J. Estatística Sem Matemática para Psicologia: usando SPSS para Windows. Porto Alegre: Artmed, 2006.

DURAND, A. C. et al. ED patients: how nonurgent are they? Systematic review of the emergency medicine literature. American Journal of Emergency Medicine, França, v. 29, n. 3, p. 333-345, mar. 2011a.

DURAND, A. C. et al. Be careful with triage in emergence departments: interobserver agreement on 1578 patients in France. BMC Emergency Medicine, Paris, v. 11, n. 19, p. 110, oct. 2011b.

FANHANI, H. R. et al. Consumo de medicamentos por idosos atendidos em um centro de convivência no noroeste do Paraná, Brasil Consumo de medicamento por idosos. Revista Brasileira de Geriatria e Gerontologia, Rio de Janeiro, v. 10, n. 3, p. 301-304, dez. 2007.

FRANCISCO, P. M. S. B.; DONALISIO, M. R.; LATTORRE, M. R. D. O. Internações por doenças respiratórias em idosos e a intervenção vacinal contra influenza no Estado de São Paulo. Revista Brasileira de Epidemiologia, Campinas, v. 7, n. 2, p. 220-227, 2004.

GARCÍA, P. O.; ARTERO, C. C. Priorities for cardiovascular health in the Americas: key messages for policymakers. Washington: Pan American Health Organization, 2011.

GENTILE, S. et al. Nonurgent patients in the emergency departments? A French formula to prevent misuse. BMC Health Services Research, França, v. 10, n. 66, p. 1-6, mar. 2010.

INSTITUTO BRASILEIRO DE GEOGRAFIA E ESTATÍSTICA (IBGE). Expectativa de vida. 2013. Disponível em: <http://teen.ibge.gov.br/noticias-teen/7827-expectativa-de-vida>. Acesso em: 1 maio 2014.

LUNDELIN, K. et al. Knowledge of stroke warning symptoms and intended action in response to stroke in Spain: a nationwide population-based study. Cerebrovascular Diseases, Barcelona, v. 34, n. 2, p. 161-168, sep. 2012. 
MENDIS, S.; PUSKA, P.; NORRVING, B. Global Atlas on Cardiovascular Disease Prevention and Control. Geneva: World Health Organization, 2011.

MOREIRA, M. M. Envelhecimento da população brasileira. 1997. Tese (Doutorado em Saúde). Universidade Federal de Minas Gerais, Belo Horizonte, 1997.

NASCIMENTO, M. R. Feminização do envelhecimento populacional: expectativas e realidades de mulheres idosas quanto ao suporte familiar. In: WONG, L. R. 0 envelhecimento da população brasileira e o aumento da longevidade: subsídios para políticas orientadas ao bem-estar do idoso. Belo Horizonte: UFMG/Cedeplar/ABEP, 2001. p. 191-218.

OLIVEIRA, G. N. et al. Perfil da população atendida em uma unidade de emergência referenciada. Revista Latino-Americana de Enfermagem, Ribeirão Preto, v. 19, n. 3, p. 1-9, maio/jun. 2011.

PAN AMERICAN HEALTH ORGANIZATION. Technical Reference Document on Noncommunicable Disease Prevention and Control. Washington: Pan American Health Organization, 2011.

RODRIGUES, R. A. et al. Política nacional de atenção ao idoso e a contribuição da enfermagem. Revista Texto e Contexto em Enfermagem, Florianópolis, v. 16, n. 3, p. 536545, jul. 2007.

RODRIGUES, P. C. O. et al. Distribuição espacial das internações por asma em idosos na Amazônia Brasileira. Revista Brasileira de Epidemiologia, Rio de Janeiro, v. 13, n. 3, p. 523532, set. 2010.

SILVEIRA, R. E.; SANTOS, A. S.; FERREIRA, L. A. Impacto da morbi-mortalidade e gastos com suicídio no Brasil de 1998 a 2007. Revista de pesquisa: cuidado é fundamental, Rio de Janeiro, v. 4, n. 4, p. 3033-3042, out./dez. 2012.

SILVEIRA, R. E. et al. Gastos relacionados a hospitalizações de idosos no Brasil: perspectivas de uma década. Einstein, São Paulo, v. 11, n. 4, p. 514-520, dez. 2013.

SIMONS, D. A. Avaliação do perfil da demanda na unidade de emergência em Alagoas a partir da municipalização da saúde e do programa Saúde da Família. Recife. 2008. $160 f$. Tese (Doutorado em Saúde Pública). Fundação Osvaldo Cruz, Recife, 2008.

SIQUEIRA, A. B. et al. Impacto funcional da internação hospitalar de pacientes idosos. Revista de Saúde Pública, São Paulo, v. 38, n. 5, p. 687-694, maio 2004.

SOUZA, M. F. M. et al. Cardiovascular disease mortality in the Americas: current trends and disparities. Heart, Washington, v. 98, p. 1207-1212, oct. 2012.

VERAS, R. P.; LOURENÇO, R. Formação humana em Geriatria e Gerontologia. Rio de Janeiro: UnATI/UERJ, 2006.

VERAS, R. P. Experiências e tendências internacionais de modelos de cuidado para com o idoso. Ciência \& Saúde Coletiva, Rio de Janeiro, v. 17, n. 1, p. 231-238, jan. 2012. 